\title{
Investigación: Diseño, construcción y validación de una escala para medir los factores psicosociales y la sintomatología orgánica en el área laboral $\left.\right|^{5}$
}

\author{
Frank R. Fraticelli Toro \\ PhD. Psicología Industrial Organizacional \\ Ponce Health Science University, Ponce, Puerto Rico \\ Public Health Program \\ Correo electrónico: ffraticelli@psm.edu
}

Roberto O. González Valles

PhD. Psicología Industrial Organizacional Pontificia Universidad Católica de Puerto Rico Correo electrónico: roberto_gonzalez@pucpr.edu

\author{
Ana Fernanda Uribe Rodríguez \\ PhD. Psicología \\ Universidad Pontificia Bolivariana \\ Correo electrónico: anafernanda.uribe@upb.edu.co
}

Ingrid Moreno Cedeño

PhD. Psicología Industrial Organizacional

Pontificia Universidad Católica de

Puerto Rico Ponce, Puerto Rico

Correo electrónico: ingridmoreno@pucpr.edu

Juan C. Orengo Valverde

PhD. Metodología de la Investigación Ponce Health Science University, Ponce, Puerto Rico

Public Health Program

Correo electrónico: jorengo@psm.edu
Recibido: $17 / 05 / 2017$

Evaluado: $13 / 07 / 2017$

Aceptado: 04/08/2017

\section{Resumen}

El objetivo principal de esta investigación fue el diseño, construcción y validación de una escala para medir los factores psicosociales y la sintomatología orgánica en el área laboral. Como marco conceptual se utilizó la Teoría Demandas y Control (Karasek, 1979). El diseño del estudio fue no experimental, transversal. Se contó con una muestra de 363 participantes de tres empresas privadas y una del sector gubernamental en Puerto Rico. La escala fue sometida a un panel de diez jueces expertos para determinar validez de contenido (RVC de > .62); posteriormente, se administró a la muestra. El valor del coeficiente de Alpha de Cronbach de .78 determinó la consistencia interna del instrumento. La validez de constructo fue realizada mediante un análisis de factores, el cual determinó como adecuados las dimensiones y los reactivos. El desarrollo de la escala para medir los factores psicosociales y su efecto en la sintomatología resulta ser una herramienta útil como una estrategia de intervención temprana.

Palabras clave

Escala, factores psicosociales, sintomatología orgánica.

5 Para citar este artículo: Fraticelli, F.R., González, R.O., Uribe, A.F., Moreno, I., \& Orengo, J.C. (2018). Investigación: Diseño, construcción y validación de una escala para medir los factores psicosociales y la sintomatología orgánica en el área laboral. Informes Psicológicos, 18(1), pp. 95-112 http://dx.doi.org/10.18566/infpsic.v18n1a05 


\section{Research: Design, construction and validation of a scale to measure psychosocial factors and organic symptomatology in the workplace}

\section{Abstract}

The main objective of this research was the design, construction and validation of a scale to measure the psychosocial factors and the organic symptomatology in the workplace. The study was non-experimental and crosssectional. It included a sample of 363 participants from three private companies and one from the government sector in Puerto Rico. The scale was submitted to a panel of ten expert judges in order to determine its content validity and, subsequently, it was administered to the sample. The construct validity of the scale was made by an analysis of factors, which determined the suitability of the dimensions and reagents. The development of the scale for measuring the psychosocial factors and their effect on the symptoms proves to be a useful tool as an early intervention strategy.

Keywords

Scale, psychosocial factors, organic symptomatology.

\section{Pesquisa: projeto, construção e validação de uma escala para medir os fatores psicossociais e a sintomatologia orgânica na área laboral}

\section{Resumo}

0 objetivo principal desta pesquisa foi 0 desenho, a construção e a validação de uma escala para medir os fatores psicossociais e a sintomatologia orgânica na área laboral. 0 desenho do estudo foi não experimental e transversal. Teve-se uma amostra de 363 participantes de três empresas privadas e uma do setor governamental no Porto Rico. A escala foi submetida a um painel de dez juízes especialistas para determinar a validade do conteúdo e posteriormente foi administrada a amostra. A validação da construção foi feita a traves de uma analise de fatores, 0 que determinou como adequadas as dimensões e os reagentes. 0 desenvolvimento da escala para medir os fatores psicossociais e seu efeito na sintomatologia revela-se como uma ferramenta útil como estratégia de intervenção precoce.

Palavras chave Escala, fatores psicossociais, sintomatologia orgânica. 


\section{ntroducción}

Cuando se habla de trabajo, el término puede definirse de múltiples maneras, sin embargo, generalmente es explicado como la inversión consciente e intencional de una determinada cantidad de esfuerzo con el fin de producir bienes, incrementar la elaboración de productos o desarrollar la realización de servicios con los que se puede satisfacer algún tipo de necesidad humana (Blanch, 1996). Así mismo, Peiró (1987, citado por Díaz, 1998), define el trabajo como:

un conjunto de actividades que, retribuidas o no, tienen un carácter productivo y creativo, permiten obtener, producir o prestar determinados bienes, productos o servicios mediante el uso de técnicas, instrumentos, materias o informaciones, de tal manera que quien las ejerce ha de aportar energías, habilidades, conocimientos y otros diversos recursos a cambio de algún tipo de compensación material, psicológica y/o social (p. 24).

Según Uribe (2013), es de esperarse que el trabajo genere ingresos para satisfacer las prioridades básicas y mejorar las condiciones de vida de las personas. Para Charria, Sarsosa y Arenas (2011), "a nivel psicológico el trabajo es fuente de interacción con otros, permite satisfacer unas necesidades de pertenencia, genera satisfacción y puede constituirse en fuente de crecimiento o ejercer una influencia potencialmente negativa sobre el individuo" (p. 381).
A pesar de las ventajas o los beneficios que se le suelen atribuir al trabajo, ciertas tareas o situaciones pueden tener efectos psicológicos muy nocivos para el individuo. Algunas condiciones de trabajo en el ámbito laboral suelen resultar en un aumento de estrés considerable y ello, a su vez, puede desembocar en distress psicológico o en otro tipo de daño al empleado, como, por ejemplo, la quemazón en el trabajo, la ansiedad, ciertas molestias físicas y hasta el deseo continuo de renunciar. Según Martín \& Góngora (2002), "la evolución de la actividad laboral ha traído consigo cierta mejoría de la calidad de vida de los trabajadores, aunque también es responsable de la aparición de una serie de efectos negativos en la salud de estos" (p. 5).

Para Kalimo, El-Batawi y Cooper (1988), la exposición a ese estrés psicosocial en los espacios laborales va acompañada en las últimas décadas de una serie de problemas de salud, entre los que se pueden incluir los trastornos del comportamiento y las enfermedades psicosomáticas. Desde el año 1974, los países miembros de la Organización Mundial de la Salud están prestando mayor atención a la cuestión de los factores psicosociales y a la relación que guardan estos con la salud y con el desarrollo humano. En lo que respecta a la salud de la población trabajadora, los autores determinaron que existe una interconección entre determinados factores psicosociales y el estado de salud del trabajador, pudiendo contribuir a causar y a agravar una enfermedad y, a su vez, a influir en los resultados de las medidas implementadas para la curación y rehabilitación del empleado.

Hay diferentes aproximaciones, tanto teóricas como conceptuales, con 
respecto a los factores de riesgo psicosociales. Mansilla-Izquierdo (2012) y Paz (2014) se refieren a estos como las condiciones presentes en una situación laboral, que están directamente relacionadas con las condiciones ambientales (agentes físicos, químicos o biológicos), con la organización (procedimientos y métodos de trabajo), con las relaciones entre los trabajadores, con el contenido del trabajo y la realización de las tareas. Tales factores pueden afectar al individuo tanto en su salud física y psicológica como en su desempeño laboral.

De acuerdo con Rodríguez (2009):

Los factores psicosociales de riesgo se presentan durante la interacción del individuo con su medio laboral. Esa aseveración lleva a concluir que prácticamente ninguna persona que trabaje estará exenta de resultar afectada por ellos. En ese medio ambiente de trabajo se suelen mezclar los componentes genéticos, psicológicos o sociales, con diferentes circunstancias que pueden afectar la salud y el bienestar de los trabajadores. Los factores psicosociales y organizacionales del trabajo, como formas de condiciones sociales, son circunstancias que se generan en los empleos y que a menudo tienden afectar a la salud laboral de la persona, tanto de forma positiva como negativamente. La cultura, el liderazgo o el clima organizacional pueden generar excelentes o pésimas condiciones de trabajo con consecuencias ventajosas - adversas para la salud de los trabajadores (p. 131).
Todos los factores psicosociales, cuando incrementan la probabilidad de afectar negativamente a la salud o al bienestar de los trabajadores, son considerados como factores de riesgo (actúan como factores desencadenantes de tensión y de estrés laboral). Desde esta perspectiva se puede concluir que los factores psicosociales de riesgo o de estrés serían definidos como factores organizacionales que manifiestan una alta probabilidad de desencadenar efectos negativos sobre la salud de la persona (Moreno \& Báez, 2010, p.8)

Para Rosario et al. (2014), los factores psicosociales de riesgo son aquellos aspectos que tienen que ver con la concepción, la organización y la gestión del trabajo, así como con el contexto social y ambiental en los cuales se lleva a cabo el mismo. Dichos factores tienen el potencial de causar daños físicos, psíquicos o sociales en los trabajadores. Como ejemplo de estos factores psicosociales se pueden mencionar: "la carga de trabajo, el poco control o poca autonomía laboral, el conflicto de roles, las altas demandas de trabajo, las limitaciones organizacionales o la supervisión y/o liderazgo inefectivo, entre otros" (p. 99)

Estudios en diversas partes del mundo proveen base científica acerca de los factores psicosociales que permean en el ambiente laboral y los efectos que estos tienen en el empleado. Yang, Haldeman, Lu, y Baker (2016) realizaron un estudio que tenía como objetivo principal estimar la prevalencia de dolor de espalda baja y su asociación a un conjunto de factores de riesgo que emergen del lugar de trabajo. Entre sus hallazgos más 
sobresalientes de este estudio se destacó que la prevalencia de dolor de espalda baja fue de un 25.7\% para todos los trabajadores (24.5\% para los hombres y $27.1 \%$ para las mujeres). Además, los trabajadores expuestos a un imbalance familia - trabajo, ambiente laboral hostil o inseguridad laboral tenían una prevalencia mayor de dicha condición.

Lesuffleur, Chastang, Sandret y Niedhammer (2014) investigaron la asociación entre los factores psicosociales del trabajo y el ausentismo de los empleados debido a enfermedad o problemas de salud. Como resultado del trabajo de campo se encontró que, tanto para los hombres como para las mujeres, el pobre reconocimiento, los cambios de turno de trabajo, el acoso psicológico y el abuso verbal resultaron ser los factores de riesgo más relevantes para motivarlos a ausentarse al trabajo.

Así mismo, Hinkka, Kuoppala, Väänänen y Lamminpää (2013) realizaron un estudio en el que midieron la asociación entre factores psicosociales del trabajo y las ausencias por enfermedad, por accidentes ocupacionales y por licencias de enfermedad. Entre los hallazgos más sobresalientes, los autores reportan que la retroalimentación frecuente por parte de los supervisores, las buenas oportunidades para el crecimiento mental, el buen clima de trabajo de equipo, la alta apreciación por parte de supervisores y la alta gerencia están íntimamente asociados a la disminución en el riesgo de ausencias por enfermedad. En el mismo estudio se encontró que la buena comunicación en el trabajo fue asociada con una disminución en la violencia hacia el trabajador, mientras que la elevada presión en el trabajo estaba relacionada con un alto riesgo de accidentes laborales.

En este mismo orden, Ishizaki et al. (2013) Ilevaron a cabo un estudio que tuvo como propósito identificar las características psicosociales del trabajo asociadas con largos períodos de ausencia por enfermedad en una muestra de empleados varones. Entre los hallazgos más importantes se reportó que un alto apoyo por parte del supervisor es asociado significativamente con la disminución en la razón de licencias por enfermedad. La ambigüedad en los roles tiende en gran medida a motivar a los empleados para que incurran en ausencias prolongadas. Los resultados en el estudio sugieren que el apoyo del supervisor en el área de trabajo puede resultar en una reducción de las ausencias por enfermedad.

En Puerto Rico, Rosario et al. (2014) evaluaron la relación entre la salud cardiovascular, los factores de riesgo psicosociales en el trabajo y el bienestar psicológico o físico (muestra de 660 personas empleadas en diferentes organizaciones de Puerto Rico). Entre los resultados, los autores establecen que los factores psicosociales se correlacionan de forma significativa, pero inversa, con la salud cardiovascular. De igual forma, los niveles de depresión y los de ansiedad se correlacionaron también de modo inverso a la salud cardiovascular. Todos los factores de riesgo psicosociales estudiados se correlacionan de forma inversa con el bienestar psicológico y físico del empleado. Además, encontraron que los síntomas físicos y el desprestigio personal parecen predecir la salud cardiovascular, mientras que el acoso psicológico, las limitaciones organizacionales, la carga de trabajo, la incivilidad y la autonomía laboral pueden 
diagnosticar los niveles de ansiedad del empleado. Por último, concluyen que la incivilidad laboral y el entorpecimiento del progreso parecen predecir los niveles de depresión de estos participantes.

Por lo expuesto anteriormente, se toma como marco teórico para la realización de la investigación el Modelo de Demandas y Control, esquema creado e implantado por Robert Karasek (1979). Éste fue desarrollado en aquellos entornos de trabajo en los cuales los estresores resultaban ser crónicos. Dicho modelo se basa en las características psicosociales del trabajo y el nivel de control del trabajador sobre ellas (Saute, Murphy, Hurrel \& Lev, 1997). Según Vega (2001), Karasek observó que los efectos del trabajo en la salud y el comportamiento parecían ser resultado de la combinación de las demandas psicológicas laborales y de las características estructurales del trabajo.

Es de suma importancia mencionar que en Puerto Rico existen estudios dirigidos a investigar los riesgos psicosociales y ciertas condiciones específicas. Ejemplo de ello se evidencia en la investigación realizada por Sánchez (1985) para investigar el estrés situacional en los niveles de ansiedad entre guardias cadetes y guardias probatorios de la Policía de Puerto Rico. Además, Anqueira (1997) investigó el síndrome de agotamiento y el ambiente de trabajo en una muestra de maestros de escuela elemental y Meléndez y Andújar (1997) analizaron los niveles de estrés que experimentan las enfermeras profesionales en instituciones hospitalarias en el área metropolitana de San Juan, Puerto Rico. Así mismo, el realizado por Rosario, González y Rovira (2011) que investigaron la relación entre el acoso psicológico ("mobbing"), los comportamientos contraproducentes y las emociones en el lugar de trabajo. También está disponible el trabajo de Ferreira (2013) que lleva por título "Factores del ambiente laboral y su relación con el turnover en enfermería". Como se puede observar, ninguna de estas investigaciones se ha enfocado en medir los factores de riesgos psicosociales y la sintomatología orgánica en el empleado.

Por lo anteriomemte expuesto, es relevante el desarrollar un instrumento que vaya dirigido a medir los factores de riesgo psicosociales y la sintomatolgía orgánica. En este sentido, Hernández, Fernández y Baptista (2006) sostienen que los instrumentos de medición pierden validez y confiabilidad cuando son llevados de una cultura a otra sin pasar por un proceso de validación. Por otro lado, Tornimbeni, Pérez y Olaz (2008) establecen que el uso de un instrumento en un contexto cultural diferente al original genera diversas dificultades. El idioma, la familiaridad con los estímulos del instrumento (ítems) y las diferentes características de las muestras de estandarización son fuentes de posibles sesgos. Por último, los autores establecen que en el ensamblaje (assemb/y), el instrumento original se modifica tan profundamente que prácticamente se transforma en un nuevo test o instrumento. Fernández, Pérez, Alderete, Richaud y Fernández (2010) establecen que:

adaptar pruebas psicológicas con el viejo procedimeinto de traducirlas textualmente y construir normas estadísticas adecuadas a la nueva población, basándose en el viejo modelo psicométrico aditivo probabilístico, es completamente inadecuado y desaconsable. En general, lo más perinente sería 
construir nuevas pruebas que operacionalicen exactamente el concepto, atributo o procesos que el investigador desea estudiar dentro de su contexto teórico y cultural, y siguiendo los principios psicométricos adecuados (p. 68).

Por todo esto, el propósito de la presente investigación fue desarrollar, construir y validar una escala para medir los factores psicosociales y la sintomatología orgánica en los empleados puertorriqueños. Con la misma, se busca recopilar información confiable para el proceso de investigación en las organizaciones y, asimismo, poder ofrecer recomendaciones de acuerdo a los hallazgos.

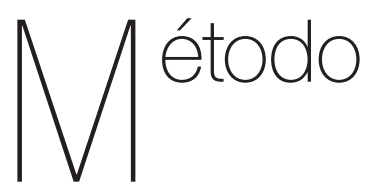

\section{Diseño de la investigación}

Para que la siguiente investigación pudiera llevarse a cabo se utilizó una metodología cuantitativa, mediante un diseño no experimental. Al utilizar este tipo de metodología, el investigador recolecta datos numéricos de los objetos, fenómenos o participantes, que estudia y analiza mediante procedimientos estadísticos (Hernández et al., 2006). El diseño no experimental se realiza sin manipulación deliberada de las variables. Ello se logra a través de la observación de los fenómenos, tal y como ocurren en su contexto natural para que después sean analizados.
El diseño del presente trabajo investigativo incluyó un enfoque transversal, en el cual se recolectan datos en un tiempo único. Dicho enfoque tuvo como propósito describir variables y analizar su incidencia e interrelación en un momento dado (Toro \& Parra, 2006).

\section{Población y participantes}

En la presente investigación se distribuyeron 380 cuestionarios, de los cuales se lograron recuperar 363 (95\%). Los mismos se distribuyen de la siguiente manera: 299 pertenecientes a la empresa privada y 64 pertenecientes al sector público. La muestra fue seleccionada aleatoriamente (todos los elementos tienen la misma probabilidad de ser elegidos). Ese tipo de muestreo puede realizarse de distintas maneras y una de ellas es el muestreo simple, es decir, se extraen todos los individuos al azar de una lista que es el marco de la encuesta (Casal \& Mateu, 2003).

Para poder participar de la investigación, las personas debían cumplir con los siguientes criterios de inclusión: ser hombre o mujer mayor de 21 años, llevar laborando en la agencia o compañía seleccionada (pública o privada) un tiempo no menor a 6 meses, ya sea a jornada parcial o completa y que el empleado sea exento o no exento. Entre los criterios de exclusión se consideraron que el trabajador sea menor de 21 años, que lleve laborando un tiempo menor de 6 meses o que la persona labore por contrato (persona con convenio verbal o escrito que brinda sus servicios a la agencia, pero éste no se considera parte de la empresa, 
y no recibe los beneficios de un empleado bonafide).

\section{Instrumentos}

Para fines de la presente investigación se diseñaron dos instrumentos, ambos autoadministrables. El primero de ellos consistió en una hoja para recopilar los datos sociodemográficos, para determinar cuál era la composición de la población. El segundo instrumento fue la escala para medir los factores psicosociales y la sintomatología orgánica.

Esta parte constó de las siguientes dimensiones: 1) Ambiente Laboral: oficina o área de trabajo con mucho frío o calor, iluminación del área, interrupciones en el área laboral, flexibilidad para ejecutar el trabajo, trabajo en equipo, sobrecarga de trabajo; 2) Sintomatología Orgánica: molestias físicas al llegar al hogar, insomnio, molestias gastrointestinales, dolor de cabeza y hombros, cambios en los estados de ánimo; y 3) Supervisión efectiva: objetivos y descripción del puesto por parte del supervisor, conflicto al momento de recibir órdenes, retroalimentación efectiva por parte del supervisor, canales de comunicación, supervisor como modelo a seguir.

Para aplicar esta escala, las opciones de respuesta fueron registradas mediante un Formato Likert. La adjudicación de puntuaciones de acuerdo a dicho formato era como sigue: 5 - totalmente de acuerdo, 4 - de acuerdo, 3 - indeciso, 2 - en desacuerdo y 1 - totalmente en desacuerdo. Los reactivos se sometieron a un panel de diez jueces expertos en el tema para que, de esta manera, se pueda determinar correctamente la validez de contenido, según el Método Lawshe (1975). A través de la técnica de jueces expertos lo que se buscaba era analizar los reactivos en términos cualitativos (Cohen \& Swerdlik, 2001).

De acuerdo con Lawshe (1975), para un panel compuesto por 10 jueces, el valor mínimo de razón de contenido o RVC es de .62. Por lo tanto, si el reactivo obtuviera un valor menor a éste, no debería ser incluido en la versión final del instrumento. Los jueces debían poseer competencia cultural, entiéndase de Puerto Rico, y del ambiente laboral. Además de esto, cada uno debió poseer, como parte de su preparación académica, un grado de maestría o doctorado en el campo de la Psicología Industrial Organizacional o en el de los Recursos Humanos.

Una vez recogidos los cuestionarios debidamente contestados, se procedió a cuantificar y luego a interpretar y analizar los datos recolectados. Con el fin de realizar diversos análisis estadísticos, se empleó el programa computarizado Statistical Package for Social Science (SPSS), versión 23.0. Además de determinar la confiabilidad mediante la aplicación del Alpha de Cronbach, se examinó también la validez del constructo mediante la utilización de un análisis de factores.

Ramada, Serra y Delclós (2013) establecen que el coeficiente Alpha de Cronbach permite cuantificar el nivel de confiabilidad de una escala y además es la medida ponderada de las correlaciones entre las premisas o ítems que forman parte de la escala. McMillan y Schumacher (2005), Oviedo y Campo-Arias (2005) y Frías-Navarro (2014), coinciden en que el valor mínimo aceptable del 
Alpha de Cronbach debe ser .70 y que un valor mayor o igual a .60, puede ser catalogado como cuestionable (puede ser aceptable tomando en consideración esa limitación). Sin embargo, Corral (2010) establece que un Alpha de Cronbach mayor e igual a .61 se puede considerar aceptable ya que posee una magnitud catalogada como alta. Para precisar correctamente la medida de determinación de adecuación de la muestra se utilizaron las pruebas Kaiser-Meyer-Okin (KMO), cuyo valor debe ser mayor de .05. Del mismo modo, se realizó la prueba de Esfericidad con una significancia de .000 .

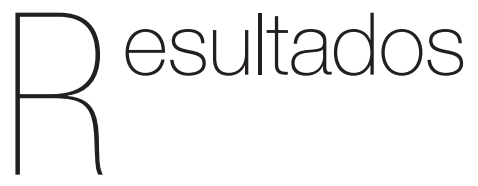

\section{Análisis datos sociodemográficos}

De los 363 cuestionarios recuperados se pudo obtener la siguiente información: el $65.3 \%$ de los participantes eran del género femenino y el $34.7 \%$ del masculino. El $44.6 \%$ de la muestra tenían una edad entre 41 a 50 años y un $24 \%$ con 51 años o más, lo que implica una población adulta. Además, los datos reflejan que los que respondieron cuentan vasta experiencia en el ámbito laboral ya que el $42.4 \%$ llevaba 16 años o más en la agencia o empresa en la que laboraban. Con respecto al tipo de empresa en la que se desempeñaban, el $82.4 \%$ de la muestra provenía de la empresa privada.

En términos de puesto que ocupan, los resultados obtenidos reflejan que la mayoría de los participantes estaban en una posición de ventas, con un 31.7\%, seguido de una posición de carácter administrativo, con un $24.2 \%$. Al analizar los años en el puesto de trabajo se observó que el 25.1\% tenían entre 2 a 5 años en su posición actual, seguido de un 19\% entre 11 a 15 años.

\section{Análisis de validez de contenido}

De acuerdo con McMillan \& Schumacher (2005), la validez de contenido trata de probar que el instrumento incluye una muestra de elementos suficiente y representativa del universo que constituye el rasgo, característica o dimensión la cual se pretende medir. De un listado inicial compuesto por 65 reactivos, 43 obtuvieron un puntaje mayor a lo estimado por el Método Lawshe.

\section{Análisis de confiabilidad}

El análisis de confiabilidad indica la consistencia interna que puede tener un instrumento. Se seleccionó el procedimiento de consistencia interna Alpha de Cronbach para estimar parámetro. En la presente investigación el coeficiente de confiabilidad reflejó un valor de .76 con las respuestas de los 39 reactivos. Por tanto, se demuestra la confiabilidad del instrumento. Esto después de evaluar las dimensiones y se suprimieron los ítems cuya correlación era negativa y/o cerca de cero (en la dimensión de Ambiente Laboral). 
Una vez realizado el análisis de confiabilidad de las 39 premisas, se obtuvo el Alpha de Cronbach de cada dimensión. Los resultados de las dimensiones de ambiente laboral, sintomatología orgánica y supervisión efectiva fueron: .618 (8 ítems), .839 (22 ítems) y .795 (9 ítems), respectivamente. Esto muestra que la dimensión sintomatología orgánica fue la que obtuvo mayor confiabilidad con un .839, mientras que la de ambiente laboral obtuvo la fiabilidad menor.

\section{Análisis factorial y validez de constructo}

De acuerdo con Díaz de Rada (2002), un requisito importante para que tenga sentido el análisis factorial es que las variables estén altamente correlacionadas. Para verificar esta condición, se empleó la prueba de esfericidad de Barlett y el índice de adecuación muestral KMO (Kaiser-Meyer-Olkin). El resultado de la significancia de la prueba de esfericidad de Bartlett $(p<.000)$, demuestra que se rechaza la hipótesis nula a un nivel de significación de un 5\%. Por lo tanto, tiene sentido realizar el análisis factorial.

Por otro lado, Díaz de Rada (2002) indicó que la medida de adecuación muestral del KMO (Kaiser-Meyer-Olkin) es otro índice que determina si es posible el análisis factorial. Éste es un índice que permite comparar las magnitudes de los coeficientes de correlación obtenidos, con las magnitudes de correlación parcial. Cuando dicho índice está comprendido entre 6 y 1, es indicado hacer el análisis factorial. En este caso, el índice de KMO obtenido fue .775, por lo que se procedió a realizar el análisis factorial. La prueba de esfericidad de Bartlett fue de .000.

De igual forma, se analizó la varianza total explicada de los reactivos de la escala. Los mismos 10 componentes son significativos y explican el $79 \%$ de la varianza, debido a que poseen un porcentaje de varianza mayor de $1 \%$.

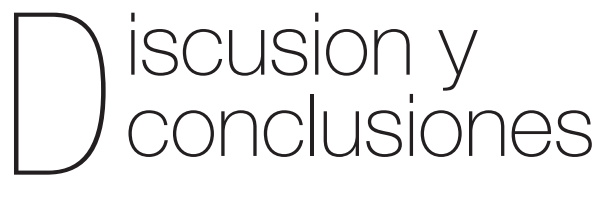

El objetivo principal en esta investigación fue el diseño, construcción y validación de una escala para medir los factores psicosociales y la sintomatología orgánica en el área laboral. Basado en la revisión de literatura, la exposición a los riesgos psicosociales es un tema de relevancia para la salud ocupacional, que puede desembocar en: enfermedades músculo esqueléticas, consecuencias fisiológicas a nivel hormonal, del sistema nervioso central, sistema cardiovascular, entre otros. Otro de los efectos de la exposición a nivel psicológico muy comunes son los problemas como: la irritación, tensión, depresión, disminución de la concentración y memoria, dificultad en la toma de decisiones y variación del rendimiento laboral, ya sea por nivel alto o nivel bajo de estrés (Uribe, 2013).

Es de suma importancia recalcar que, para construir dicho instrumento, se tomó como base la Teoría de Modelo de Demandas y Control de Robert Karasek. 
Este modelo constituye una de las construcciones teóricas más relevantes e influyentes dentro de la investigación desarrollada sobre el entorno psicosocial del trabajo, el estrés y la enfermedad. Al mismo tiempo, es uno de los modelos que aporta mayor evidencia científica para explicar los efectos de las condiciones de trabajo sobre la salud y el comportamiento, los cuales son el resultado de la combinación de dos dimensiones: las demandas o exigencias psicológicas que implica el trabajo para la persona y la capacidad de control como recurso moderador fundamental (Gil-Monte, López-Vilchez, Llorca-Rubio, \& Sánchez, 2016, p. 9).

La construcción y validación de un instrumento propone medir el objetivo que se halla trazado en la investigación. En una investigación de esta naturaleza los aspectos más importantes son la validez y la confiabilidad. De acuerdo con Lawshe (1975), para un panel compuesto por 10 jueces, el valor mínimo de razón de contenido o RVC es de .62. En esta investigación, 43 reactivos obtuvieron un valor mayor a lo propuesto por Lawshe.

Los hallazgos sugieren la confiabilidad entre factores psicosociales del trabajo y sintomatología orgánica en el empleado. De acuerdo con Herrans (2000), confiabilidad es el grado de presición que tienen los resultados que se derivan mediante la administración de un examen a un individuo o grupo de individuos.

Ésta se obtuvo por el análisis del Alpha de Cronbach que, basado en McMillan \& Schumacher (2005), se trata de un coeficiente de confiabilidad aceptable si refleja un valor mayor de .70. El instrumento obtuvo un coeficiente de Alpha de Cronbach de .76. En adición se evaluó la confiabilidad por cada una de las dimensiones en el instrumento (Ambiente laboral, Sintomatología orgánica y Supervisión efectiva), y éstas obtuvieron un coeficiente de .618, .839 y .795, respectivamente. Se puede observar que la dimensión Ambiente Laboral no logró un coeficiente mayor a .70; de acuerdo con Corral (2010), un coeficiente mayor o igual a .610 es aceptable. Las variables que fueron suprimidas con correlaciones totales negativas y/o cercanas a cero fueron: Considero que tengo autonomía para ejecutar mi trabajo (-.328), Mi trabajo es repetitivo (-.769), Mi puesto ofrece oportunidades de aprender cosas nuevas (-.361) y Mi área de trabajo me ofrece buena iluminación, ventilación adecuada y/o aire acondicionado (-.670).

Entre los hallazgos más significativos se encuentran que el instrumento presentó validez y confiabilidad adecuada, además de que sus variables se ajustaron de manera satisfactoria al modelo propuesto mediante el análisis de factores. De acuerdo con estos resultados, podemos determinar que el instrumento cuenta con las propiedades psicométricas necesarias para ser administrado en población laboral de Puerto Rico. Esto es de gran significado ya que, según Herrans (2000), existe una gran necesidad de desarrollar instrumentos de medición que se ajusten a las características de la población. Además, se está aportando con una herramienta que permite medir cómo los factores psicosociales inciden en la sintomatología orgánica del trabajador. Una vez administrado ofrece al empleador una idea de cómo está organizada su compañía, qué cosas se deben evaluar más 
a fondo para tomar medidas correctivas, prevenir el ausentismo y accidentes laborales. Y, a su vez, aumentar la producción y proteger al recurso más importante que es el recurso humano.

\section{Limitaciones}

Validar un cuestionario para la población puertorriqueña es una tarea ardua debido a la cantidad de participantes requeridos para estos fines. La muestra fue escogida en cinco corporaciones, cuatro privadas y una del sector público, y su participación fue voluntaria. Como todos los trámites para tener acceso a la muestra se hizo a través del Departamento de Recursos Humanos; algunas personas declinaban de su participación y se pudo percibir un poco de cautela al participar, a pesar de garantizar su confidencialidad. De igual forma, se les dio un tiempo límite a los participantes para completar el cuestionario. En dos de las agencias, el investigador entregó y recogió los cuestionarios el mismo día. Esto se puede considerar como una limitación ya que los participantes pudieron interpretar cómo hay que hacerlo con rapidez. Se puede considerar el no haber distribuido los reactivos de manera más equitativa entre las tres dimensiones como una limitación.

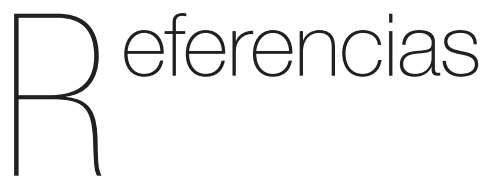

Agra, B. (2006). Las Enfermedades del trabajo: Nuevos Riesgos Psicosociales y su Valoración en el derecho de la protección social. Ministerio del Trabajo y Asuntos Sociales, España. Recuperado de http:// www.seg-social.es/prdi00/groups/public/ documents/binario/100517.pdf

Anastasi, A. \& Urbina, S. (1997). Psychological Testing. 7th Edition. New York: Prentice Hall Editors

Anqueira, J. \& Andújar, C. (1997). El síndrome de agotamiento y el ambiente de trabajo en una muestra de maestros de escuela elemental. (Tesis inédita de maestría). Universidad Carlos Albizu, San Juan, PR. Recuperado de: http://athena.sju.albizu. edu/athcgi/athweb.pl

Badii, M., Guillen, J., Landeros, J., \& Cerna, E. (2011). Análisis y aplicación de muestreo multietápico, estimación de submuestreo y muestreo de respuesta aleatoria. International Journal of Good Conscience, 6(2), 88-95

Bakker, A. \& Demerouti, E. (2013). La teoría de las demandas y los recursos laborales. Journal of Work and Organizational Psychology, 29, 107-115

Bartolomé, M., Fernández, C., Prieto, S., \& Sánchez, V. (s.f.). Riesgos psicosociales emergentes en el trabajo. Universidad de León, España. Recuperado de http://www. ruct.uva.es/pdf/Revista\%208/8414.pdf

Blanch, J.M. (1996). Psicología del Trabajo. Psicología Social Aplicada, Tercera Edición, Madrid: McGraw Hill Editores

Bonett, D. (2002). Sample Size Requirements for Testing and Estimating Coefficient Alpha. Journal of Educational and Behavioral Statistics 27(4), 335-340 
Buela, G. \& Sierra, J. (1997). Manual de evaluación psicológica; Fundamentos, técnicas y aplicaciones. Primera Edición. Madrid: Siglo Veintiuno de España Editores, S.A.

Cambell, J., Quick, J. Nelson, D., \& Hurrell, J. (1997). Preventive Stress Management in Organizations. Recuperado de http:// www.apa.org/pubs/books/4317920.aspx

Cano, A. (2002). Estrés laboral. Sociedad Española para el estudio del estrés y la ansiedad. Recuperado de http:// pendientedemigracion.ucm.es/info/seas/ estres_lab/index.htm

Cardarelli, A., Campos, D., Gutiérrez, D., Ansolega, E., Miranda, G., Pérez, J., Toro, J., Caroca, L., Constanza, M., Moya, M., Badal, M., Majluf, M., Garrido, P., Elizalde, S., \& Ruiz, V. (2013). Protocolo de Vigilancia de Riesgos Psicosociales en el Trabajo. Departamento de Salud Ocupacional, Subsecretaria de Salud Pública, Ministerio de Salud, República de Chile. Recuperado de www.campusprevencionisl.cl/.../ vigilancia/riesgos_psicosociales.pdf

Casal, J. \& Mateu, E. (2003). Tipos de Muestreo. Revista Epidemiológica de Medicina Preventiva, 1, 3-7.

Catalá, F. (2000). La Economía de Puerto Rico, 1898-1998. Recuperado de http:// economia.uprrp.edu/Boletin_de_\%20 Economia.html

Cohen, R.J., \& Swerdlik, M. E. (2001). Pruebas y evaluación psicológicas: Introducción a las pruebas y a la medición. México: McGrawHill

Confederación de Empresarios de Málaga, España (2013). Guía de Prevención de
Riesgos Psicosociales en el Trabajo. Recuperado de http://www.cemmalaga.es/portalcem/novedades/2013/ CEM_guia_riesgos_psicosociales_ interactivopdf

Córdoba, E. (2010). Factores psicosociales y su influencia en el bienestar laboral. Facultad de Psicología de la Universidad de Buenos Aires. Recuperado de http://23118.psi.uba.ar/academica/ carrerasdegrado/psicologia/informacion_ adicional/obligatorias/040_trabajo1/ cdcongreso/CD/TRABAJOS\%2OLIBRES/ SALUD\%20MENTAL/3.pdf

Córdoba, E. \& Petit, L. (2012). Construcción de un Instrumento de Bienestar Psicológico Laboral. Trabajo presentado en el IV Congreso Internacional de Investigación y Práctica Profesional en Psicología. Facultad de Psicología -Universidad de Buenos Aires, Buenos Aires. Recuperado de https://www.aacademica.org/000072/392.pdf

Corporación del Fondo del Seguro del Estado (2012). Estadísticas y Utilización de servicios. Documento: Tomo II (Aprobado). Recuperado de http://web.fondopr.com/ es/la-corporacion/estadisticas

Corral, Y. (2010). Diseño de cuestionarios para recolección de datos. Revista Ciencias de la Educación, 20(36), 52-168

Charria, V., Sarsosa, K., \& Arenas, F. (2011). Factores de riesgo psicosocial laboral: métodos e instrumentos de evaluación. RevistaFacultad NacionaldeSaludPública, 29(4), 380-391. Recuperado de http:// web.a.ebscohost.com/ehost/pdfviewer/ pdfviewer? vid=9\&sid=c1567277-e53e4703-bfd8-05e45dca5e24\%40sessionm gr4004\&hid $=4214$ 
Departamento del Trabajo y Recursos Humanos (2012). Estadísticas relacionadas con la Administración de Seguridad y Salud Ocupacional en Puerto Rico. Estado Libre Asociado de Puerto Rico. Recuperado de http://www.trabajo.pr.gov/det_estadistica. asp?cnt_id=359

De Vellis, R. (2003). Sale Development Theory and Applications. Applied Social Research Methods Series. Second Edition. USA: Sage Publications Inc.

Díaz de Rada, V. (2002). Técnicas de análisis multivariante para investigación social y comercial. Madrid: Rama

Díaz, L. (1998). Psicología del Trabajo y las Organizaciones. Concepto, Historia y Método. Departamento de Psicología Cognitiva, Social y Organizacional de la Universidad de La Laguna, España. Recuperado de idiazvi.webs.ull.es

Durán, M. (2010). Bienestar psicológico el estrés y la calidad de vida en el contexto laboral. Revista Nacional de administración, 1(1), 71-84

Fernández, A., Pérez, E., Alderete, A., Richaud, M., \& Fernández, L. (2010). ¿Construir o adaptar test psicológicos? Diferentes respuestas a una cuestión controvertida. Artículo metodológico. Revista Evaluar, 10, 60-74. Recuperado de https://revistas. unc.edu.ar/index.php/revaluar/article/ download/459/428

Ferreira, F. (2013). Factores delambiente laboral y su relación con el turnover en enfermería. (Tesis inedita de maestría). Universidad Metropolitana, San Juan, PR. Recuperado de http://www.anagmendez.net/umet/pdf/ biblioteca_tesissalud_ferreiraroccaf2013. pdf
Frías-Navarro, D. (2014). Aproximación al uso del coeficiente alfa de Cronbach. Recuperado de http://www.uv.es/friasnav/ ApuntesSPSS.pdf

Gil-Monte, P., López-Vilchez, J., Llorca-Rubio, J., \& Sánchez, J. (2016). Prevalencia de riesgos psicosociales en personal de la Administración de Justicia de la Comunidad Valenciana (España). Revista de Psicología, 22(1), 7-19. Recuperado de http://www.redalyc.org/ pdf/686/68646348001.pdf

Gil, P. (2012). Riesgos psicosociales en el trabajo y salud ocupacional. Revista Peruana de Medicina Experimental y de Salud Pública, 29(2), 237- 41. Recuperado de http://www.scielo.org.pe/pdf/rins/ v29n2/a12v29n2.pdf

Gold, L. \& Shuman, D. (2009). Evaluating Mental Disability in the Workplace. Model, Process and Analysis. USA: Springer Dordrecht Heidelberg Editores

Guerrero, J. \& Pastrana, M., (2009). Guía de Prevención de Riesgos psicosociales en el trabajo. Departamento de Asistencia Técnica para la Prevención de Riesgos Laborales. Recuperado de http://www. empleo.gob.es/itss/web/index.html

Gutiérrez, A. \& Viloria, J. (2014). Riesgos Psicosocialesyestrésenelambientelaboral. Revista Salud Uninorte, Barranquilla, 30(1), 1-3. Recuperado de http:// web.a.ebscohost.com/ehost/pdfviewer/ pdfviewer?vid=2\&sid=c1567277-e53e4703-bfd8-05e45dca5e24\%40sessionm gr4004\&hid $=4214$

Harris, C., Eisen, E A., Dale, AM., Evanoff, B., Hegmann, KT., Thiese, M. Kapellush, J., Garg, A., Burt, S., Bao, S., Silverstein, B., 
Gerr, F., Merlino, L., \& Rempel, D. (2013). Personal and workplace psychosocial risk factors for carpal tunnelsyndrome: apooled study cohort. Journal of Occupational and Environmental Medicine, 70(8), 529-37.

Hinkka, K., Kuoppala, J., Väänänen, I., \& Lamminpää, A. (2013). Psychological work factors and sick leave, occupational accident and disability pension: a cohort study of civil servants. Journal of Occupational and Environmental Medicine, 55(2), 191-197.

Herrans, L. (2000). Psicología y Medición. El desarrollo de pruebas psicológicas en Puerto Rico. Segunda edición. México: McGraw Hill.

Ishizaki, M., Kawakami, N., Yamada, Y., Nakagawa, H., Morikawa, Y., Work Stress and Health Cohort Study Group (2013). A prospective study of psychological work characteristic and long sick leave of Japanese male employees in multiple workplaces. Industrial Health, 51(4), 398-405

Juárez, A. (2007). Factores psicosociales, estrés y salud en distintas ocupaciones: un estudio exploratorio. Recuperado de http://www.imbiomed.com.mx/1/1/ articulos. php? method=showDetail\&id_ articulo $=47115 \&$ id_seccion $=3005 \&$ id ejemplar=4771\&id_revista $=179$.

Kalimo, R., El-Batawi, M., \& Cooper, C. (1988). Los factores psicosociales en el trabajo y su relación con la salud. Organización Mundial de la Salud. Recuperado de http://whqlibdoc.who.int/ publications/1988/9243561022_spa.pdf

Kasl, S.V. (1984). Stress and health. Annual Review of Public Health, 5, 319-341.
Landy, F. \& Conte, J. (2004). Psicología Industrial. Introducción a la psicología industrial y organizacional. Primera edición en español. México: McGraw Hill, Editores.

Lawshe, C. (1975). A Quantitative Approach to Content Validy. Personnel Psychology, 28, 563-575

Lesuffleur, T., Chastang, J.F., Sandret, N., \& Niedhammer, I. (2014). Psychological factors at work and sickness absence: results from the French national SummerSurvey. American Journal of Industrial Medicine, 57(6), 695-708.

Linares, J. (2014). Diseño, construcción y validación de una escala para medir ergonomía en el área laboral (Tesis de Doctorado). Pontificia Universidad Católica de Puerto Rico.

McMillan, J.H., \& Schumacher, S. (2005). Investigación educativa (5ta edición). México: Pearson Addison Wesley.

Mansilla-Izquierdo, F. (2012). Manual de Riesgos Psicosociales en el trabajo: Teoría y Práctica: Cómo influyen los factores de riesgos psicosociales en el trabajo y su prevención. Recuperado de http://www. psicologia-online.com/ebooks/riesgos/

Mansoor, M., Fida, S., Nasir, S., \& Ahmad, Z. (2011). The impact of job stress on employee job satisfaction: A study on telecommunication sector of Pakistan. Journal of Business Studies Quartely, 2(3), 50-56

Martín-Baró, I. (2010). Acción e Ideología. Psicología Social desde Centroamérica. Universidad Centroamericana "José Simeón Cañas": UCA Editores. 
Martín, M. \& Góngora, J. (2002). Factores Psicosociales, identificación de situaciones de riesgo. Instituto Navarro de Salud Laboral. Recuperado de http://www.ual. es/GruposInv/Prevencion/evaluacion/ procedimiento/F.\%20Factores\%20 Psicosociales.pdf

Matsudaira, K., Isomura, T., Miyoshi, K., Okazaki, H., \& Konishi, H. (2014). Risk Factors for low back pain and katakori: a new concept. Japanese Journal of Clinical Medicine, 72(2), 244-50

Meléndez, E. \& Andujar, C. (1997). Niveles de estrés que experimentan las enfermeras profesionales en el desempeño de sus funciones en los servicios generales e intensivo en instituciones hospitalarias en el área metropolitana de San Juan Puerto Rico (Tesis inédita de maestría). Universidad Carlos Albizu, San Juan, PR. Recuperado de http://athena.sju.albizu. edu/athcgi/athweb.pl

Melero, L., Pérez, M., Cruz, M., Melero, A., \& Palacios, B. (2011). Las consecuencias de la Organización del Trabajo en la Salud Laboral en la Empresa: estudio de las variables en la aparición de riesgos sociales. UGT-CEC: Universidad de Salamanca.

Morales, P., Urosa, B., \& Blanco, A. (2003). Construcción de escalas de actitudes tipo Likert. Una guía práctica. Madrid: La Muralla.

Moreno, B. \& Báez, C. (2010). Factores y Riesgos Psicosociales, formas, consecuencias, medidas y buenas prácticas. Universidad Autónoma de Madrid. Recuperado de http://www.insht. es/InshtWeb/Contenidos/Documentacion/ PUBLICACIONES\%2OPROFESIONALES/ factores\%20riesgos\%20psico.pdf
NIOSH (1999). El estrés en el trabajo. Publicación Número 99-101. Recuperado de http://www.cdc.gov/spanish/niosh/ docs/99-101_sp/\#lugar

OIT/OMS (1984). Factores Psicosociales en el Trabajo: Naturaleza, incidencia y prevención. Informe del Comité Mixto OITOMS sobre Medicina del Trabajo, novena reunión Ginebra, 18-24 de septiembre de 1984. Recuperado de http://biblioteca. uces.edu.ar/MEDIA/EDOCS/FACTORES_ Texto.pdf

Organización Mundial de la Salud (2010). Ambientes de trabajo saludables: un modelo para la acción. Para empleadores, trabajadores, autoridades normativas y profesionales. Recuperado de http://apps.who.int/iris/bitstre am/10665/44317/1/9789243599311_spa. pdf?ua=1

OSHA, (2015). Occupational Safety and Health Administration. United States Department of Labor. Recuperado de www.osha.gov/ workers-spanish

Oviedo, H. \& Campo-Arias, A. (2005). Metodología de Investigación y lectura crítica de estudios. Aproximación al uso del coeficiente de alfa de Cronbach. Revista Colombiana de Psiquiatría, 34(4), 572-580

Pando, M., Angels, M., Arrellano, G., \& Saraz, S. (2006). Los factores psicosociales en el trabajo. Recuperado de http://sedi. oas.org/ddse/documentos/rial/sso_peru/ Documentos\%20aportados\%20por\%20 Ios\%20participantes/Factores $\% 20$ Psicosociales \%20y\%20Salud\%20 Mental\%20en\%20el\%20Trabajo.pdf

Paz, M. (2014). Factores Psicosociales. Recuperado de http://www.facmed.unam. 
mx/deptos/salud/censenanza/spivst/ spiv/factorespsico.pdf Pekkarinen, L., Elovainio, M., Sinervo, T., Heponiemi, T., Aalto, A.M., Noro, A., \& Finne- Soveri, H. (2013). Job demands and musculoskeletal symptoms among female geriatric nurses: the moderating role of psychosocial resources. Journal of Occupational Health Psychology, 18(2), 211-219.

Pérez, A. \& Muriel, I. (2007). El absentismo laboral, en relación con el estrés, la compensación, beneficios y sus implicaciones en términos de costos: comparación entre Estados Unidos y China. (Tesis de Maestría). Universidad Interamericana, Ponce, Puerto Rico.

PROSHA (2015). Normas y Reglamentos. Puerto Rico OSHA. Recuperado de www. trabajo.pr.gov/prosha

Ramada, J., Serra, C., \& Delclós, G. (2013). Adaptación cultural y validación de cuestionarios de salud: revisión y recomendaciones metodológicas. Revista de Salud Pública de México, 27(1), 57-66.

Rodríguez, M. (2009). Factores Psicosociales de Riesgo Laboral: ¿Nuevos tiempos, nuevos riesgos? Observatorio Laboral Revista Venezolana, 2(3) 127-141. Recuperado de http://www.redalyc.org/ articulo.oa?id=219016838007

Román, J., Gelpí, J., Cano, A., \& Catalina, C. (2009). Manual para Combatir el Estrés Laboral. Madrid: Editorial y Producción Arteanima, S.L. Recuperado de http:// www.ibermutuamur.es/IMG/pdf/ MANUAL_COMO_COMBATIR_EL_EST RESLABORAL_-_web.pdf

Rosario, E., Rovira, L. V., Rodríguez, A., Rivera, B. E., Fernández, L. N., López, R. S., ... \&
Ortiz, M. A. (2014). La salud cardiovascular y su relación con los factores de riesgo psicosociales en una muestra de personas empleadas en Puerto Rico. Revista Puertorriqueña de Psicología, 25(1), 98116. Recuperado de http://pepsic.bvsalud. org/scielo.php?pid

Saute, S., Murphy, L., Hurrell, J., \& Lev, L. (1997.). Factores Psicosociales y de la Organización. En J. Stellman (Eds.). Enciclopedia de Salud y Seguridad Ocupacional (pp. 34-77). Vol. 1. Suiza: Oficina de Trabajo Internacional. Recuperado de http://www.insht.es/ InshtWeb/Contenidos/Documentacion/ TextosOnline/EnciclopediaOIT/tomo2/34. pdf

Savio, S. (2008). El Síndrome del Burn Out: Un Proceso de Estrés Laboral Crónico. Hologramática, 8(1), 121-138. Recuperado de http://www.cienciared.com.ar/ra/doc. php?n=829

Spector, P. (2008). Industrial and organizational psychology. Research and practice. 5th Edition. USA: Wiley \& Sons Editors

Tornimbeni, S., Pérez, E., \& Olaz, F. (2008). Introdución a la psicometría. 1ra Edición. Buenos Aires: Paidós Editores.

Toro, I.D. \& Parra, R.D. (2006). Método y conocimiento: Metodología de la investigación. Medellín: Fondo Editorial Universidad EAFIT.

Uribe, A. (2013). Factores Psicosociales en las Organizaciones. Bucaramanga: Universidad Pontificia Bolivariana.

Urien, B. \& Osca, A. (2012). Estresores de rol, norma de orientación a la tarea y satisfacción laboral: un estudio longitudinal. 
Revista de Psicología el Trabajo y de las Organizaciones, 28(3), 171-181

Vandergrift, J.L., Gold, J.E., Hanlon, A. \& Punnett, L. (2012). Physical and psychological ergonomic risk factors for low back pain in automotive manufacturing workers. Journal of Occupational and Environment Medicine, 69(1), 29-34

Vega, M. (2001). Riesgo psicosocial: el modelo demanda-control-apoyo social. Ministerio de trabajo y asuntos sociales España. Instituto nacional de Seguridad e Higiene en el Trabajo. Recuperado de http://www.insht.es/InshtWeb/Contenidos/ Documentacion/FichasTecnicas/NTP/ Ficheros/601a700/ntp_603.pdf
Warner, R. M. (2008). Applied Statistics: from bivariate through multivariate techniques. Estados Unidos: Sage Publication.

Yang, H., Haldeman, S., Lu, M. L., \& Baker, D. (2016). Low back pain prevalence and related workplace psychosocial risk factors: a study using data from the 2010 National Health Interview Survey. Journal of manipulative and physiological therapeutics, 39(7), 459-472. 